\title{
Study of angular responses of mixed amplitude-phase holographic gratings: shifted Borrmann effect
}

\author{
L. Carretero, R. F. Madrigal, A. Fimia, and S. Blaya \\ Departamento de Ciencia y Tecnología de Materiales, Universidad Miguel Hernández, Av. del Ferrocarril s/n Apdo. 03202. Edificio \\ Torrevaillo, Elche (Alicante), Spain \\ A. Beléndez \\ Departamento de Física, Ingeniería de Sistemas y Teoría de la Senal, Universidad de Alicante, Apdo. 99, E-03080, Alicante, Spain
}

Received October 10, 2000

\begin{abstract}
We present theoretical results for angular responses of transmitted and diffracted beams in mixed amplitude-phase holographic gratings. Experimental results for gratings recorded in photographic emulsions and developed without a bleaching bath, with diffraction efficiencies of $>20 \%$, are also presented. The model shows an angular shift between minimum transmittance and maximum diffraction efficiency when both index modulation and absorption coefficient modulation are present. Also, the Borrmann effect was found outside the Bragg angle. This method can be extended to a study of the mechanism of image formation in materials such as bacteriorhodopsin and azo-dye-doped polymers that exhibit both types of modulations (index and absorption). (C) 2001 Optical Society of America

OCIS codes: $090.7330,160.2900$.
\end{abstract}

The main parameters of holographic gratings, such as index and absorption modulations, determine the gratings' properties. They also enable us to understand the photophysical and photochemical processes that take place in the medium and consequently lead to the development of new materials that could improve the properties of those used at present. Generally, to research holographic materials we need to induce periodic deviations in their properties by light irradiation from interferential experiments.

The coupled-wave theory of Kogelnik ${ }^{1}$ has been used as a powerful tool for determination of the diffraction efficiency of a volume grating, giving us a sinusoidal spatial modulation of refractive index, absorption coefficient, or both.

We wish to consider the analysis of gratings that exhibit simultaneous refractive-index and absorption modulation. This is an important situation in the study of the physics that underlies the processes of photochemistry ${ }^{2-4}$ in the study of photorefractive media that contain bacteriorhodopsin, ${ }^{5}$ or, for example, in holographic gratings recorded in colored $\mathrm{KBr}$ crystals. ${ }^{6,7}$

Holographic materials have usually been analyzed by study of the diffraction efficiency of the grating at symmetrical geometry. We studied theoretically and experimentally the angular responses of diffracted and transmitted light (outside Bragg's angle) and found that an angular shift appears in the transmitted beam in relation to the diffracted beam when absorption and index modulation are present simultaneously. Therefore this result can be used for obtaining quantitative values of modulation index and absorption parameters of gratings.

When a complex grating is illuminated with one normalized plane wave $\mathbf{R}$ outside Bragg angle $\theta_{b}$, the nor- malized complex electric-field amplitudes for the transmitted and the diffracted waves, $\mathbf{R}$ and $\mathbf{S}$, are given by ${ }^{1}$

$$
\begin{aligned}
\mathbf{R} & =\frac{\kappa^{2}}{c_{s}\left(\gamma_{1}-\gamma_{2}\right)}\left[\frac{\exp \left(\gamma_{2} d\right)}{c_{r} \gamma_{2}+\alpha}-\frac{\exp \left(\gamma_{1} d\right)}{c_{r} \gamma_{1}+\alpha}\right] \\
\mathbf{S} & =i \frac{\kappa}{c_{s}\left(\gamma_{1}-\gamma_{2}\right)}\left[\exp \left(\gamma_{2} d\right)-\exp \left(\gamma_{1} d\right)\right] .
\end{aligned}
$$

For symmetrical geometry, $c_{r}=c_{s}=\cos \theta$, where $\theta$ is the angle of incidence of the reconstruction wave, $d$ is the thickness of the material, $\kappa=\kappa_{1}-i \kappa_{2}$ is the coupling constant, given by

$$
\kappa=\frac{\pi n_{1}}{\lambda}-i \frac{\alpha_{1}}{2}
$$

where $n_{1}$ and $\alpha_{1}$ are the amplitudes of the spatial modulation of the refractive index and the absorption constant, respectively, and $\lambda$ is the wavelength in free space. Finally, the parameters of $\gamma$ are given by

$$
\begin{aligned}
\gamma_{1,2}= & -\frac{1}{2}\left(\frac{\alpha}{c_{r}}+\frac{\alpha}{c_{s}}+i \frac{\vartheta}{c_{s}}\right) \\
& \pm \frac{1}{2}\left[\left(\frac{\alpha}{c_{r}}-\frac{\alpha}{c_{s}}-i \frac{\vartheta}{c_{s}}\right)^{2}-4 \frac{\kappa^{2}}{c_{r} c_{s}}\right]^{1 / 2},
\end{aligned}
$$

where $\vartheta$ is the dephasing factor that appears when the grating is reconstructed outside the Bragg angle and is given (for symmetrical geometry) by 


$$
\vartheta=\frac{4 \pi n_{0} \sin \theta_{b}}{\lambda}\left(\sin \theta-\sin \theta_{b}\right)
$$

where $n_{0}$ is the average refractive index of the material and $\alpha$ is the absorption constant. We obtain the diffraction and transmission efficiencies by multiplying Eqs. (1) and (2) by the conjugate complex, $R^{*}$ and $S^{*}$, respectively. After some algebraic transformations and introduction of the parameters $z_{0}$ and $\psi_{0}$ :

$$
\begin{aligned}
& z_{0}=\left[\left(\vartheta^{2}+4\left(\kappa_{1}^{2}-\kappa_{2}^{2}\right)^{2}+\left(8 \kappa_{1} \kappa_{2}\right)^{2}\right]^{1 / 2},\right. \\
& \psi_{0}=\arccos \frac{-\left[\vartheta^{2}+4\left(\kappa_{1}^{2}-\kappa_{2}^{2}\right)\right]}{z_{0}},
\end{aligned}
$$

it is possible to write analytical equations for diffraction efficiency $\left(\eta_{d}\right)$ and transmission efficiency $\left(\eta_{t}\right)$ as functions of angle $\theta$ of reconstruction:

$$
\begin{aligned}
& \eta_{d}(\theta)=\exp \left(-\frac{2 \alpha d}{\cos \theta}\right) \frac{\kappa_{1}^{2}+\kappa_{2}^{2}}{z_{0}} \\
& \times\left\{2 \cosh \left[\frac{\sqrt{z_{0}} d \cos \left(\psi_{0} / 2\right)}{\cos \theta}\right]\right. \\
& \left.-2 \cos \left[\frac{\sqrt{z_{0}} d \sin \left(\psi_{0} / 2\right)}{\cos \theta}\right]\right\}, \\
& \eta_{t}(\theta)=\frac{\exp [-(2 \alpha d / \cos \theta)]}{z_{0}} \\
& \times\left\{\left(\frac{\vartheta^{2}}{4}+\frac{z_{0}}{4}\right) 2 \cosh \left[\frac{\sqrt{z_{0}} d \cos \left(\psi_{0} / 2\right)}{\cos \theta}\right]\right. \\
& -\left(\frac{\vartheta^{2}}{4}-\frac{z_{0}}{4}\right) 2 \cos \left[\frac{\sqrt{z_{0}} d \sin \left(\psi_{0} / 2\right)}{\cos \theta}\right] \\
& +\vartheta \sqrt{z_{0}} \sin \left(\frac{\psi_{0}}{2}\right) \sinh \left[\frac{\sqrt{z_{0}} d \cos \left(\psi_{0} / 2\right)}{\cos \theta}\right] \\
& \left.-\vartheta \sqrt{z_{0}} \cos \left(\frac{\psi_{0}}{2}\right) \sin \left[\frac{\sqrt{z_{0}} d \sin \left(\psi_{0} / 2\right)}{\cos \theta}\right]\right\} \cdot
\end{aligned}
$$

As can easily be seen from Eqs. (8) and (9), when we introduce $\theta=\theta_{b} \Rightarrow \vartheta=0$, the usual Kogelnik ${ }^{1}$ equations for Bragg incidence are obtained. When the incidence is not in the Bragg angle, $\theta \neq \theta_{b} \Rightarrow \vartheta \neq 0$, angular responses of diffraction efficiency and transmitted efficiency are obtained from Eqs. (8) and (9). The first interesting case is obtained when $n_{1}=0$ (pure absorption grating) and the value of $\alpha_{1}$ is equal to (for example) $70 \%$ of $\alpha$; then, as can be seen from Fig. 1, there is an anomalous reduction in absorption called the Borrmann effect, ${ }^{8}$ and as a result the maximum transmittance and diffraction efficiency can be observed at the Bragg angle. When there is no absorption modulation, $\alpha_{1}=0$, then we obtain the classic results for phase gratings, for which the maximum diffraction efficiency is obtained at the Bragg angle where the transmitted intensity shows a minimum. In this case we have assumed that there is no overmodulation at the Bragg angle and consequently that at this angle the diffraction efficiency is maximal. A third situation is that in which $\alpha_{1}$ and $n_{1}$ are both different from 0 , so in this case we have an absorption-index mixed grating. Figure 2 shows three cases, $\alpha_{1} / \alpha=1,0.7,0.4$, from which it can be observed that the minimum transmittance is shifted in relation to the maximum diffraction efficiency. When the ratio $\alpha_{1} / \alpha$ is increased, the deviation in the minimum of transmittance from the Bragg angle also increases; the maximum deviation is obtained for the ratio $\alpha_{1} / \alpha=1$, which means that the contribution of the absorption grating has reached its limit $\left(\alpha_{1}=\alpha\right)$.

From Fig. 2, outside but near the Bragg angle it can be seen that there is a simultaneous increase of $\eta_{d}$

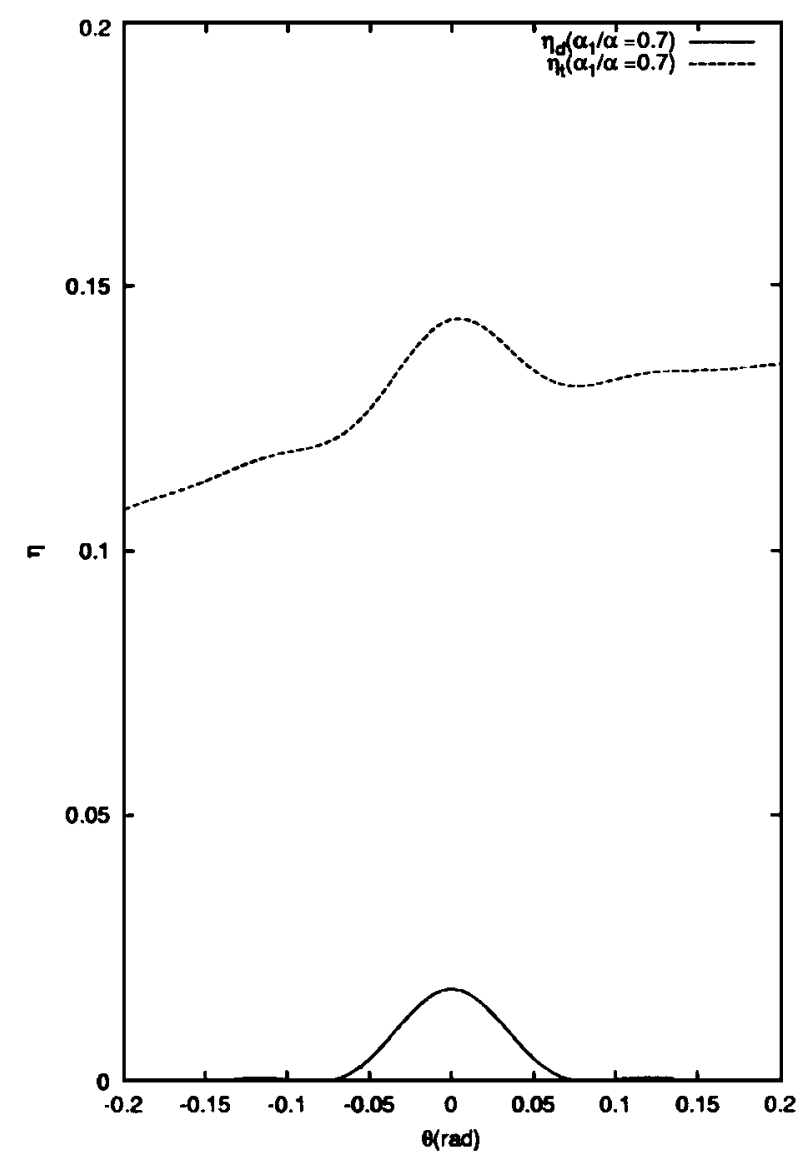

Fig. 1. Theoretical curves of the angular behavior of the normalized diffraction and transmission efficiencies of a pure absorption holographic grating recorded in symmetrical geometry with a thickness of $10 \mu \mathrm{m}, n_{1}=0, \alpha=$ $1000 \mathrm{~cm}^{-1}, \alpha_{1}=0.7 \alpha, \theta_{b}=22.5 \mathrm{deg}$, and $\lambda=633 \mathrm{~nm}$. The origin of $\theta$ is $\theta_{b}$. 


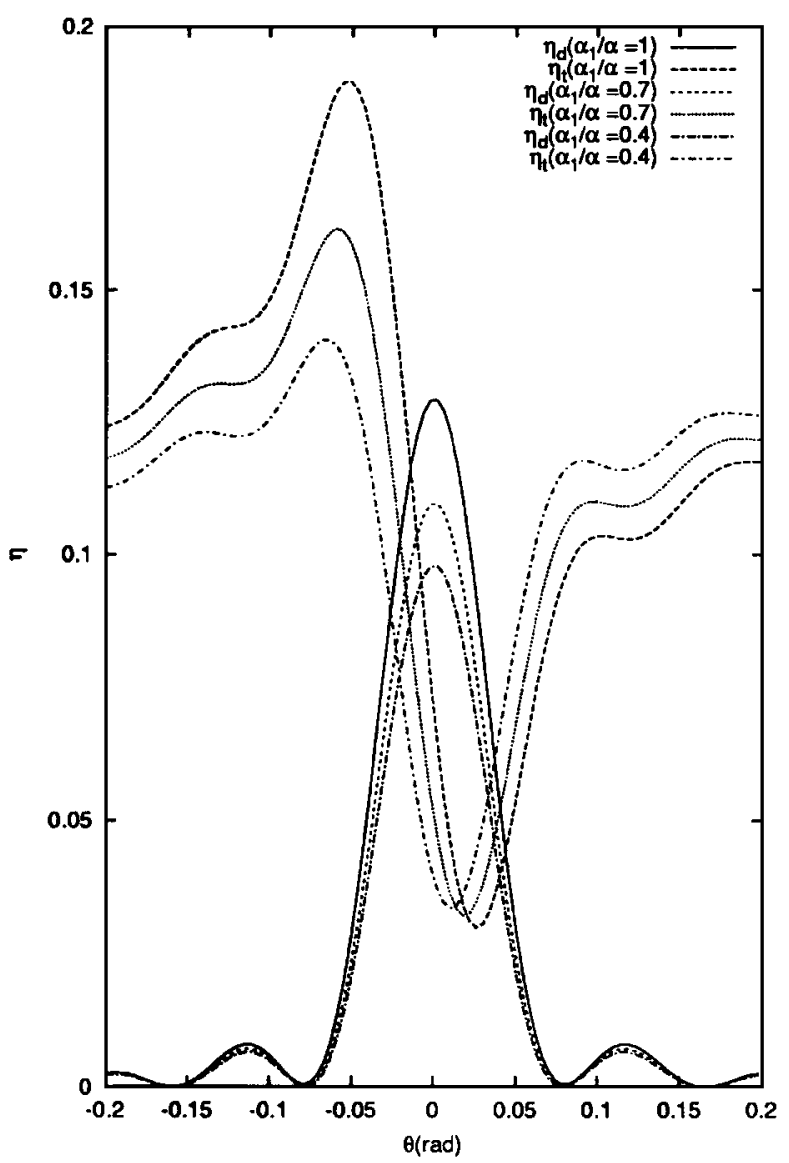

Fig. 2. Theoretical study of the effect of the modulation of absorption in the angular responses of the diffraction and transmission efficiencies of a mixed absorption-phase holographic grating recorded in symmetrical geometry with a thickness of $10 \mu \mathrm{m}, n_{1}=0.02, \alpha=1000 \mathrm{~cm}^{-1}$, $\theta_{b}=22.5 \mathrm{deg}$, and $\lambda=633 \mathrm{~nm}$. The origin of $\theta$ is $\theta_{b}$.

and $\eta_{t}$. This is behavior similar to that of the Borrmann effect (Fig. 1), although when index and absorption modulation are present the maximum for $\eta_{t}$ is shifted in relation to the maximum diffraction efficiency. As can be seen from Fig. 3, this effect can be observed in holographic gratings recorded in Agfa 8E75 HD silver halide emulsions with a spatial frequency of $\sim 1200$ lines $/ \mathrm{mm}$. When the hologram was recorded, a semiphysical development (without bleaching) process was used, ${ }^{9}$ and the resultant grating was part in phase and part in absorption, showing the advantages of both types of hologram.

The nonlinear fit of the experimental data of Fig. 3 with Eqs. (8) and (9), would give us quantitative information on $n_{1}$ and $\alpha_{1}$ parameters. As a result, we could obtain information about the effects of the developer, showing that without a bleaching bath it is possible to obtain index modulation. This situation is qualitatively evident in Fig. 3 if we take into account that a maximum diffraction efficiency of $30 \%$ is obtained, but to quantify the influence of absorption or

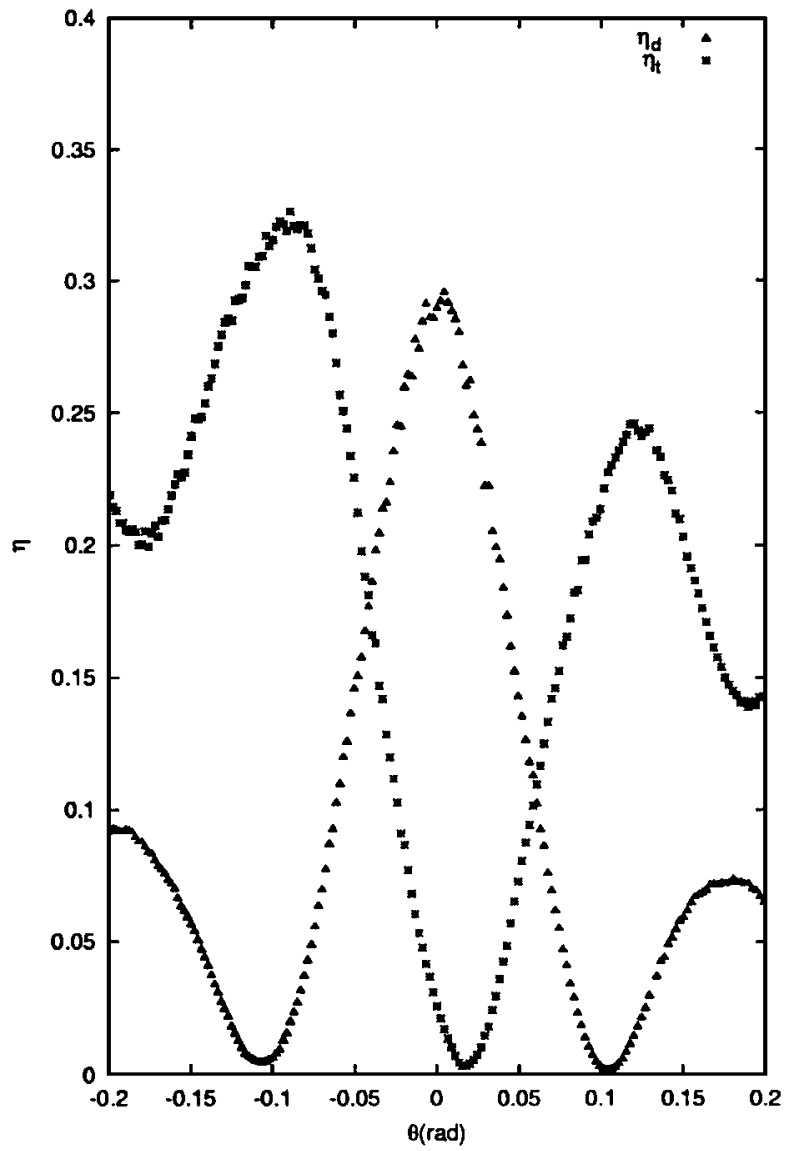

Fig. 3. Experimental curves of the angular behavior of the diffraction and transmission efficiency of a mixed absorption-phase holographic grating recorded in photographic emulsions by use of symmetrical setup with a thickness of $7 \mu \mathrm{m}, \theta_{b}=22.5 \mathrm{deg}, \lambda=633 \mathrm{~nm}$. The origin of $\theta$ is $\theta_{b}$.

index modulation on diffraction efficiency we need to study angular responses.

L. Carretero's e-mail address is luis@dite.umh.es.

\section{References}

1. H. Kogelnik, Bell. Syst. Tech. J. 48, 2909 (1969).

2. D. M. Burland, G. C. Bjorklund, and D. C. Álvarez, J. Am. Chem. Soc. 102, 7117 (1980).

3. D. M. Burland and C. Brauchle, J. Chem. Phys. 76, 4502 (1982).

4. S. Blaya, L. Carretero, R. Mallavia, A. Fimia, and R. F. Madrigal, Appl. Opt. 38, 955 (1999).

5. Y. Barmenkov and N. Kozhevnikov, Sov. Tech. Phys. Lett. 16, 28 (1990).

6. G. E. Scrivener and M. Tubbs, Opt. Commun. 10, 32 (1974).

7. O. Salminen, A. Ozols, and P. Riihola, J. Mod. Opt. 41, 1507 (1994).

8. P. Russell and L. Solymar, Appl. Phys. 22, 335 (1980).

9. A. Beléndez, R. F. Madrigal, I. Pascual, and A. Fimia, Proc. SPIE 3956, 376 (2000). 